\title{
Use of three-dimensional organoids and lung-on-a-chip methods to study lung development, regeneration and disease
}

\author{
Konstantinos Gkatzis ${ }^{1}$, Sara Taghizadeh ${ }^{2}$, Dongeun Huh ${ }^{3}$, Didier Y.R. Stainier ${ }^{1}$ \\ and Saverio Bellusci ${ }^{4,5}$ \\ Affiliations: \\ ${ }^{1}$ Dept of Developmental Genetics, Max Planck Institute for Heart and Lung Research, Bad Nauheim, Germany. \\ ${ }^{2}$ Dept of Internal Medicine, Justus-Liebig University Giessen, Giessen, Germany. \\ ${ }^{3}$ Dept of Bioengineering, University of Pennsylvania, Philadelphia, PA, USA. \\ ${ }^{4}$ Dept of Pulmonary and Critical Care Medicine, The First Affiliated Hospital of Wenzhou Medical University, \\ Wenzhou, Zhejiang, China. \\ ${ }^{5}$ Excellence Cluster Cardio-Pulmonary System, Justus-Liebig University Giessen, Giessen, Germany and \\ German Center for Lung Research (DZL).
}

\section{Correspondence:}

Konstantinos Gkatzis, Dept of Developmental Genetics, Max Planck Institute for Heart and Lung Research, Ludwigstrasse 43, 61231 Bad Nauheim, Germany.

E-mail: konstantinos.gkatzisampi-bn.mpg.de

@ERSpublications

3D organoids and lung-on-a-chip: modelling lung physiology and pathology in a dish http://ow.ly/NWCN30m0SyJ

Cite this article as: Gkatzis K, Taghizadeh S, Huh D, et al. Use of three-dimensional organoids and lungon-a-chip methods to study lung development, regeneration and disease. Eur Respir J 2018; 52: 1800876 [https://doi.org/10.1183/13993003.00876-2018]

ABSTRACT Differences in lung anatomy between mice and humans, as well as frequently disappointing results when using animal models for drug discovery, emphasise the unmet need for in vitro models that can complement animal studies and improve our understanding of human lung physiology, regeneration and disease. Recent papers have highlighted the use of three-dimensional organoids and organs-on-a-chip to mimic tissue morphogenesis and function in vitro. Here, we focus on the respiratory system and provide an overview of these in vitro models, which can be derived from primary lung cells and pluripotent stem cells, as well as healthy or diseased lungs. We emphasise their potential application in studies of respiratory development, regeneration and disease modelling. 


\section{Introduction}

The lung is a complex organ, in its most proximal part consisting of a tree-like tubular branched system (bronchiolar airways) connected to a single tracheal tube. Towards the periphery, the bronchiolar airways become progressively smaller and branch into smaller tubes (bronchioles) which terminate into alveolar regions where gas exchange takes place [1, 2]. In addition, different types of mesenchymal cells, vasculature, cartilage and neurons surround this epithelial network and contribute to lung development and homeostasis (figure 1) [1].

The complex composition and structure of the lung are established during early embryonic development. The respiratory system is specified from the anterior foregut endoderm at embryonic day (E) 9.5 in mouse (around week 4 in the human) as two primary lung buds emerge on the ventral side, distal to the rudimentary trachea surrounded by mesoderm and a vascular network [2]. During the pseudoglandular stage, which takes place between E9.5 and E16.5 in mouse (week 24 in the human), these buds undergo a process of branching morphogenesis, in which epithelial bud tips continuously bifurcate resulting in a proximal-to-distal hierarchical branched tree-like tubular network [2, 3]. It is well established that epithelial-mesodermal interactions play key roles in directing this process. Of particular interest is the mesenchyme located at the bud tips. These mesenchymal cells migrate proximally and at the level of the cleft differentiate into smooth muscle cells to stabilise the cleft formed between two daughter buds, thereby facilitating the branching process [4]. At the same time, mesenchymal-derived diffusible signals including Fgfs, Tgfb/Bmps, Wnts, Egfs and Hedgehog $(\mathrm{Hh})$ orchestrate the expansion and lineage specification of the epithelial progenitor pools located at the proximal and distal airways $[2,5]$. This developmental event in turn leads to the canalicular stage (E16.5-E17.5 in mouse and weeks 24-26 in the human) and saccular stage (E17.5 to birth or weeks 26-36 in the human), during which the distal buds become progressively narrower and the distal progenitor cells at the epithelial tips begin to differentiate into alveolar epithelial cells that populate the distal sacs, such as alveolar epithelial type 1 (AEC1) and alveolar epithelial type 2 (AEC2) cells $[6,7]$. Finally, the newly formed sacs continue to develop via a process referred to as alveolarisation, which starts at birth and continues until postnatal day (P)20 in mice, whereas in humans

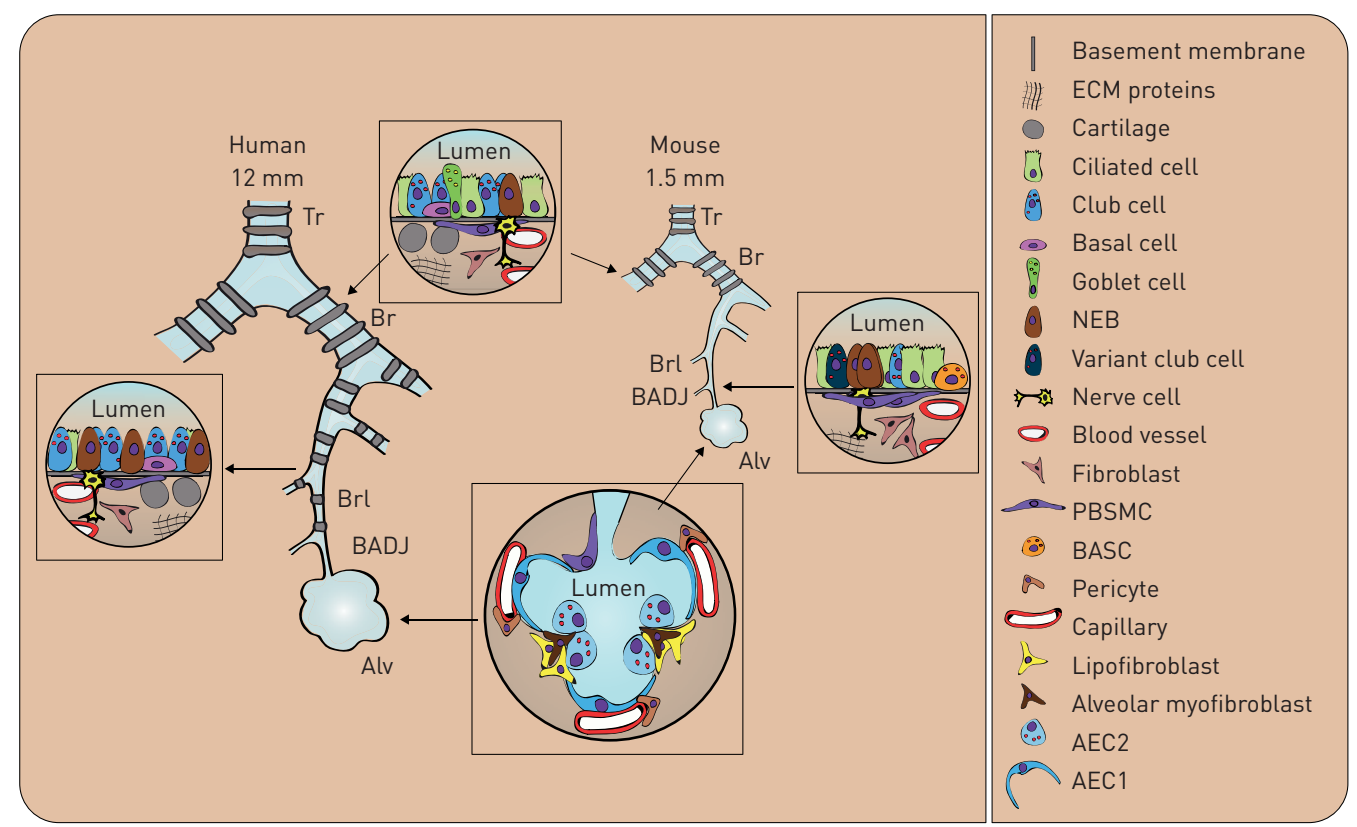

FIGURE 1 Human and mouse lung architecture. The walls of the human conducting airways consist of pseudostratified epithelium with basal, multiciliated and secretory cells surrounded by supporting smooth muscle, fibroblasts, cartilage, vasculature and neurons extending along the airways. The epithelium lining the bronchi $(\mathrm{Br})$ is predominantly composed of mucus-secreting goblet cells, scattered neuroendocrine cells and neuroendocrine bodies (NEBs), whereas the bronchiolar epithelium (Brl) is lined by club cells and an increased number of neuroendocrine cells and NEBs. In the mouse, only the pseudostratified epithelium of the trachea $(\mathrm{Tr})$ and bronchi are surrounded by cartilage. The bronchioles are lined by a simple epithelium that consists of multiciliated and club cells, a small number of neuroendocrine cells, NEBs and bronchioalveolar stem cells (BASCs). In the distal alveolar region (Alv), the epithelium is highly vascularised and composed of flat alveolar type 1 cells (AEC1), flanked by cuboidal alveolar type 2 cells (AEC2), which form a $600-\mathrm{nm}$ to $2-\mu \mathrm{m}$ thin barrier and provide an extensive surface area for gas diffusion [118-120]. BADJ: bronchoalveolar duct junction; ECM: extracellular matrix; PBSMC: parabronchial smooth muscle cell. 
alveolarisation begins in week 36 and continues for up to 3 years. The sacs undergo a number of subdivisions by the ingrowing crests (septae) which consist of myofibroblasts, lipofibroblasts, endothelial cells and pericytes, resulting in the formation of the alveoli [2]. These critical events have prompted studies into the molecular mechanisms that mediate lung development, as well as the elaboration of compatible in vitro and ex vivo tools.

In conditions of adult lung homeostasis, cell turnover is normally very low and the existing airway progenitor cells are quiescent. Remarkably, numerous animal studies have shown that a number of progenitor cells have the capacity to proliferate and differentiate into one or more cell types in response to a variety of injuries including exposure to infectious agents, tobacco smoke, high levels of oxygen and the chemotherapy drug bleomycin [1].

The regenerative ability of these progenitor cells has triggered the scientific community to search for drugs that could activate them in vivo or establish cell-based therapies which would enable the treatment of patients suffering from respiratory disease arising from lung injury or ageing. Unfortunately, the translational transfer of the results obtained using animal models, mostly mice, has been limited so far, and is probably due to unappreciated differences in lung anatomy and physiology between mice and humans. Altogether, these observations emphasise the unmet need for lung model systems that can complement animal models and validate the results obtained in animals to humans, as well as improve our understanding of human lung physiology, regeneration and disease.

In this review, we focus on two lung model systems, organoids and lung-on-a-chip, which will play a protagonist role in respiratory biomedical research. We discuss the applications of these approaches in the fields of stem cell differentiation, regenerative medicine, respiratory disease modelling and drug screening.

\section{What are organoids?}

Organoids are three-dimensional (3D) self-organised aggregates of multiple cell types grown within gels made of a complex mixture of different extracellular matrix (ECM) proteins, including laminin, fibronectin, collagen and heparin sulfate proteoglycans (figure 2). The main commercial gel used to grow organoids is Matrigel, a protein mixture secreted by Engelbreth-Holm-Swarm mouse sarcoma cells, which is liquid at low temperature and solidifies into a relatively soft gel at $37^{\circ} \mathrm{C}$. In addition to ECM proteins, Matrigel contains a mixture of growth factors such as transforming growth factor (TGF)- $\beta$, epidermal growth factor (EGF) and fibroblast growth factor (FGF), which can impact the differentiation and proliferation of many cell types. The ECM gels can mimic the in vivo extracellular environment in part and allow some of the cells to display characteristics of stem cells, namely self-renewal and multipotency. Once grown, these organoids can be reseeded for prolonged periods of time without changes in their karyotype. Additionally, these organoids can be cryopreserved without obvious alterations of the stem cells characteristics. When exposed to differentiating media, organoids give rise to specific cell types observed in the corresponding adult tissue in vivo $[8,9]$.

These novel 3D cultures are generated from primary stem cells harvested from mouse or human lungs, or even from progenitor cells derived from pluripotent stem cells (PSCs) (figure 2), and they have revolutionised biomedical research by enabling studies of in vitro organ development, disease modelling and cell-cell interaction (supplementary figure S1).

Critical to the respiratory field, organoids have been successfully generated from epithelial progenitor or stem cells originating from mouse embryonic lung [10-13], human embryonic lung [14, 15], mouse adult lung [16-20], human adult lung [16, 19,21], mouse (m)PSCs [22] and human (h)PSCs [23-28]. Further refinement of these approaches, with the scope of improving the differentiation efficiency, include co-culture of these epithelial progenitor cells with mesenchymal cells [20, 22, 23, 26, 29] or endothelial cells [18]. In addition to the epithelial-based organotypic cultures mentioned, mesenchymal-based 3D cultures have been generated using fetal lung tissue or hPSCs and cultured in bioreactors. This approach resulted in the formation of a structural phenotype similar to that of native alveolar regions in vivo [30].

\section{Differentiation of pluripotent stem cells into lung progenitors}

Organoids derived from PSCs require an additional step compared to primary organotypic cultures, as PSCs have to undergo directed differentiation in two-dimensional monolayer cultures using defined growth factor cocktails that attempts to mimic gastrulation, lung patterning and lineage commitment. These stepwise events use information gained from decades of animal model research and involve multiple signalling pathways that are controlled in a tight temporal and spatial manner in vivo.

For example, hPSC cultures are directed into the endodermal lineage using ActivinA, which mimics the Nodal signalling that has been shown to be critical for definitive endoderm specification in vertebrates [31]. Definitive endoderm formation is followed by acquisition of foregut identity by inhibiting both bone 


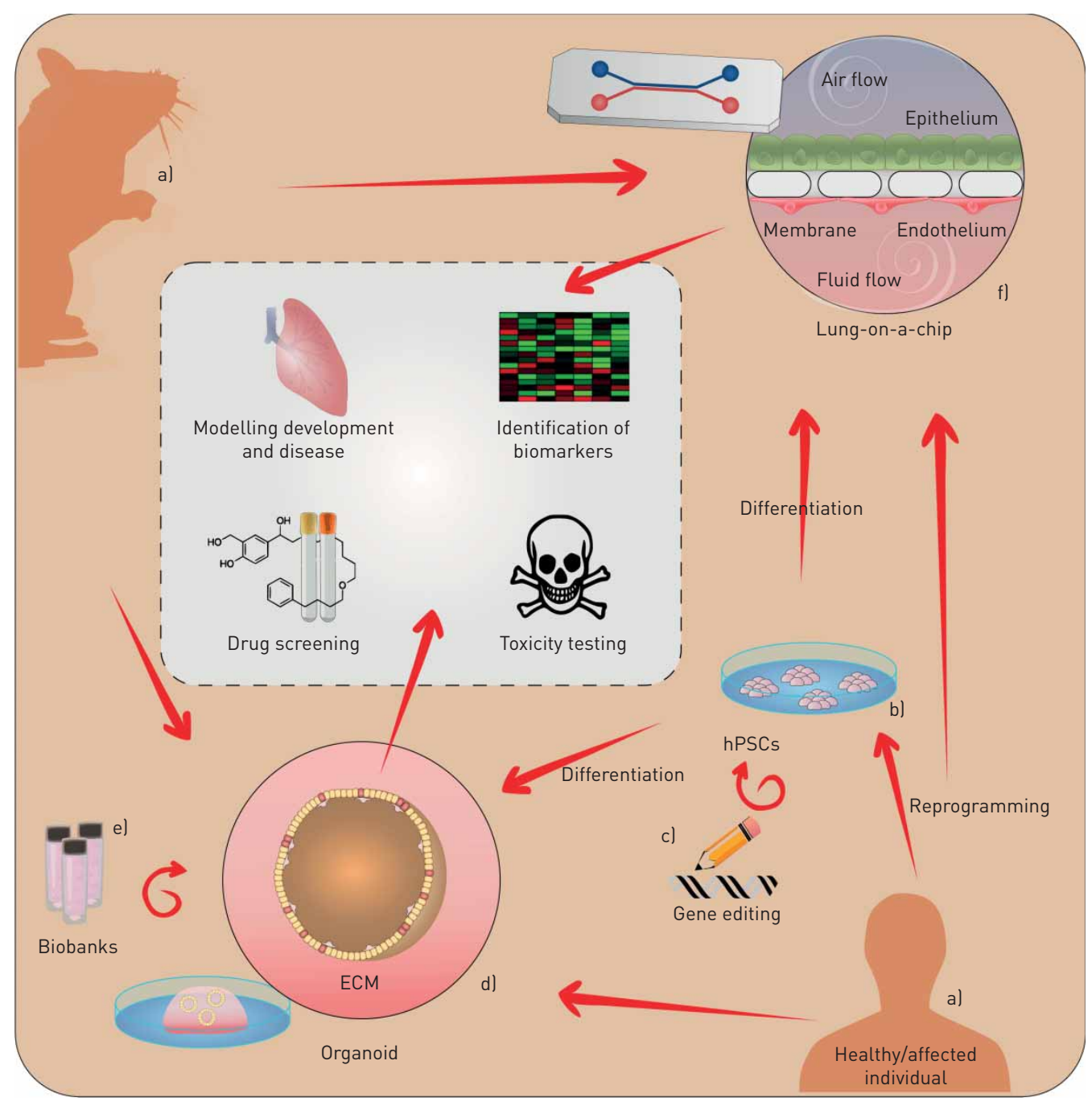

FIGURE 2 Derivation and use of organoids and lung-on-a-chip. Human and mouse organoids have been generated from stem cell populations isolated from a) healthy or diseased lung tissue and pluripotent stem cells (PSCs). b) PSCs, such as embryonic stem cells or induced pluripotent stem cells (iPSCs) can be maintained in culture for a number of passages and differentiate into derivatives of the three germ layers. iPSCs can be generated from reprogrammed somatic cells isolated from healthy individuals or people who suffer from respiratory diseases. The reprogramming process is performed by overexpressing the Yamanaka factors OCT3/4, SOX-2, KLF4 and C-MYC in somatic cells and expanding individual iPSC colonies under conditions that support PSC self-renewal $[121,122]$. c) Gene editing approaches such as the CRISPR/Cas9 technology enable the generation or correction of mutations leading to respiratory disease, providing appropriate control cells for these studies. With the development of robust differentiation protocols, iPSCs can be used to generate airway and/or alveolar epithelial cells. d) Further culture in Matrigel and specific growth factor cocktails can give rise to organoids which resemble distinct spatial regions of the lung. In addition, organoids can be grown from mouse stem cells isolated from the adult lung as well as from mouse PSCs. Depending on the origin of the stem cell population used, organoids are called tracheospheres (trachea), bronchospheres (large airways) or alveolospheres (alveolar region). e) In future, organoid biobanks of healthy and diseased lung tissues will be established. These biobanks could be a major source of organoids covering the range of genetic mutations known to lead to respiratory disorders. f) Lung-on-a-chip systems have been generated from differentiated cells, such as airway and/or alveolar epithelial cells, fibroblasts and endothelial cells derived from primary cultures or isolated from healthy or diseased human or mouse tissue. It remains to be tested whether human (h)PSC-derived cells can mimic human biology and human disease in the lung-on-a-chip approach. Both organoids and lung-on-a-chip systems are amenable to high-throughput set-ups and have immediate application to model respiratory diseases, identify new drugs able to normalise the disease phenotype, evaluate the potency and toxicity of candidate drugs and identify biomarkers of drug interactions and drug induced injury in the lung. In addition, both approaches have possible applications in personalised medicine, for instance to reveal which patients may benefit from treatment with a specific drug. ECM: extracellular matrix.

morphogenetic protein (BMP) and TGF- $\beta$ signalling pathways using Noggin or small molecule inhibitors. However, the secreted WNT inhibitors that help to maintain foregut identity in vivo are not necessary to induce foregut endoderm in vitro [23-25, 32-36]. In mouse, diffusible morphogen signals from cardiac 
and mesenchymal mesoderm orchestrate progenitor specification via activation of multiple signalling pathways [5]. Thereafter, in hPSC cultures, lung progenitors expressing NKX2.1 have been successfully generated using growth factor cocktail containing FGF, BMP, WNT, Hedgehog and retinoic acid [23-27, 32-35, 37-39]. Cell sorting methods, such as fluorescence-activated cell sorting, enable the constitution of homogeneous progenitor populations. Subsequently, these isolated cells are seeded in ECM gels to facilitate the development of an organoid culture and increase their differentiation towards conducting airway or alveolar epithelial cells. Given the unique contribution of epithelial progenitor cells to lung development and function, modelling this aspect using organoids from healthy or diseased lungs represents a powerful model for deciphering the underlying molecular mechanisms. Finally, such organotypic cultures are a suitable tool for high-throughput drug efficacy testing and toxicity effect and small-molecule screening studies.

\section{Organoids to model early stages of lung development}

The establishment of the initial proximo-distal epithelial patterning begins with the first respiratory stem cell population marked by the expression of the transcription factor Nkx2.1 in the ventral anterior foregut endoderm. In mouse, this domain is denoted by two main endodermal stem/progenitor pools marked by proximal Sox2 expression or distal Sox9 and Id2 co-expression [2, 5]. However, in contrast to mice, human progenitor cells located at the distal tips co-express SOX2 and SOX9 during pseudoglandular stage and become single SOX9 positive during the canalicular stage $[14,15,40]$. Sox $2^{+}$progenitor cells generate the conducting airway epithelium, including secretory cells, multiciliated cells, neuroendocrine cells and basal cells, and are shown to be regulated by intracellular Notch signalling [41-43]. Sox9/Id $2^{+}$progenitor cells are initially capable of generating both airway and alveolar epithelial cell types [44]. Following branching morphogenesis after E13.5, Sox $/ \mathrm{Id} 2 / \mathrm{Bmp}_{4} / \mathrm{Spc}^{+}$progenitors are restricted in their fate and only generate AEC1 and AEC2 cells [44]. In an effort to grow and maintain embryonic progenitor cells in vitro, a number of groups used serum-free organotypic cultures derived from mouse [13, 15] and human embryonic tissue [14, 15]. Following a chemical screen, Nichane et al. [13] observed that a growth factor cocktail containing Fgf9, Fgf10, Egf and inhibitors for Gsk3, Tgf- $\beta$, Rock and p38 pathways are essential for long-term mouse progenitor maintenance. Moreover, Nikolić et al. [14] were able to maintain human embryonic lung epithelial tip progenitors long-term in vitro in the presence of seven factors: EGF, FGF7, FGF10, NOG (Noggin), RSPO1 (R-Spondin 1), CHIR99021 and SB431542. Unfortunately, these conditions were not able to maintain long-term self-renewal of undifferentiated mouse lung tip epithelial progenitors in vitro, suggesting differences of functional significance between the human and mouse distal progenitor cells [14]. In contrast, MiLler et al. [15] demonstrated that FGF7, CHIR-99021 and retinoic acid support mouse and human embryonic epithelial lung tip growth and maintenance in vitro. Importantly, using the same culture conditions, they were also able to mimic human embryonic lung development in vitro by inducing hPSC-derived NKX2.1 ventral foregut cells into a tip-like progenitor state. Using hPSC derived from patients suffering from respiratory disease together with such culture conditions could benefit studies aiming to model respiratory disease in a dish.

Given the transcriptomic significant similarities of the human iPSC-derived lung bud organoids (LBOs) to the second trimester of human gestation samples, the use of hPSCs to study human lung development remains the gold standard [28]. A number of studies have reported the establishment of 3D cultures able to self-renew and generate airway epithelial cells [24, 25, 27, 28, 32-35, 45-48]. Of note, KonisHi et al. [24] used carboxypeptidase $\mathrm{M}(\mathrm{CPM})$ as a surface marker of NKX2.1 ${ }^{\text {pos }}$ ventralised anterior foregut endoderm cells (VAFECs) in order to generate proximal airway progenitor cell spheroids able to give rise to functional multiciliated cells with beating cilia. Work utilising directed airway and distal PSC differentiation revealed that the WNT pathway regulates proximodistal patterning of purified NKX2.1 lung epithelial progenitor; WNT activation using CHIR99021 promotes distal epithelial differentiation while CHIR99021 withdrawal prompts rapid proximal patterning [27].

Distal organotypic cultures have also been generated from hPSCs applying similar concepts used to derive airway cultures mentioned above $[23,25,26,28,38,39,47-50]$. PSC-derived alveolospheres are successfully generated from enriched $\mathrm{CPM}^{\text {pos }}$ VAFECs when co-cultured with human fibroblasts under alveolar-related growth factor culture conditions. Most studies have shown that a combination of dexamethasone, CHIR99021, FGF7, cAMP and IBMX is sufficient to drive alveolar differentiation and generate SFTPC ${ }^{\text {pos }}$ epithelial cells $[23,26,28,48,50]$. To derive distal epithelial cells and partly overcome the maturity limitation displayed by PSC-derived epithelial cells, some groups have employed prolonged in vitro cultures $[25,28]$, co-culture with fetal lung mesenchyme [48], seeding into acellular lung matrices [38], differentiation in a rotating bioreactor culture system [49] or transplantations under the murine kidney capsule $[28,34,51]$. Together, data from these approaches suggest that mechanical forces of the ECM, as well as signals from the extracellular matrix or from mesenchymal cells (e.g. reconstituting epithelial-mesenchymal cell interactions) all play a key role in promoting alveolar epithelial fate. 
Although the branching morphogenesis stage during embryonic lung development has been well characterised, the diversity of cellular events during the sacculation stage are still poorly understood. Using organotypic cultures derived from minced E17.5 fetal mouse lungs, MondRinos and co-workers [10, 11] demonstrated how exogenous FGF signals drive the action of diverse endogenous mediators (Sonic Hedgehog, vascular endothelial growth factor-A and tenascin-C) during de novo fetal pulmonary alveolar tissue morphogenesis.

\section{Lung homeostasis, regeneration and disease}

Lineage-tracing experiments during lung development or regeneration/repair after acute injury of distinct parts of the lung (tracheobronchial airways, bronchiolar airways or the alveoli) have allowed the identification of region-specific epithelial stem/progenitor cells (figure 3). More recently, the identity of the corresponding stromal niches allowing self-renewal and differentiation of these epithelial cells towards distinct airway and/or alveolar epithelial lineages has also been unravelled.

\section{Organoids for modelling airway regeneration and disease}

Several progenitors are also produced during lung development from the Sox2-positive (Sox $2^{\text {pos }}$ ) early endoderm progenitors. These include basal stem cells (BSCs), which express transcription factor Trp63 (p63) and cytokeratin 5 (Krt5), and are considered to be long-term stem/progenitor cells. BSCs are able to generate multiciliated and secretory epithelium of the trachea and proximal bronchiolar region during postnatal growth, homeostasis and after airway injury in adult mice (figure 3a) [16]. The first organoids derived from adult tracheal basal cells were called tracheospheres. Single mouse Krt5-GFP ${ }^{\text {pos }}$ basal cells were able to self-renew and generate luminal cells, including differentiated ciliated and secretory cells [16]. The transcriptional profile of $\mathrm{Krt} 5^{\text {pos }}$ /isolectin $\beta 4^{\text {pos }}\left(\mathrm{GSI} \beta 4^{\text {pos }}\right.$ ) cells identified two cell-surface markers, Ngfr and Itga6, which were used to isolate human lung basal cells by flow cytometry and successfully generate human tracheospheres. As with the mouse system, human tracheospheres are able to self-renew and generate luminal daughter cells [16, 52, 53]. In multiple reports from Hogan and co-workers [17, 54-57] using combined in vivo and tracheosphere approaches, the integrative role of key transcription factors and Notch, Bmp, Wnt and Il6 signalling was demonstrated during basal cell proliferation and differentiation. In addition to tracheospheres, human bronchiospheres composed of functional multiciliated cells, mucin-producing goblet cells and airway basal cells have been derived from human airway basal cells [58].
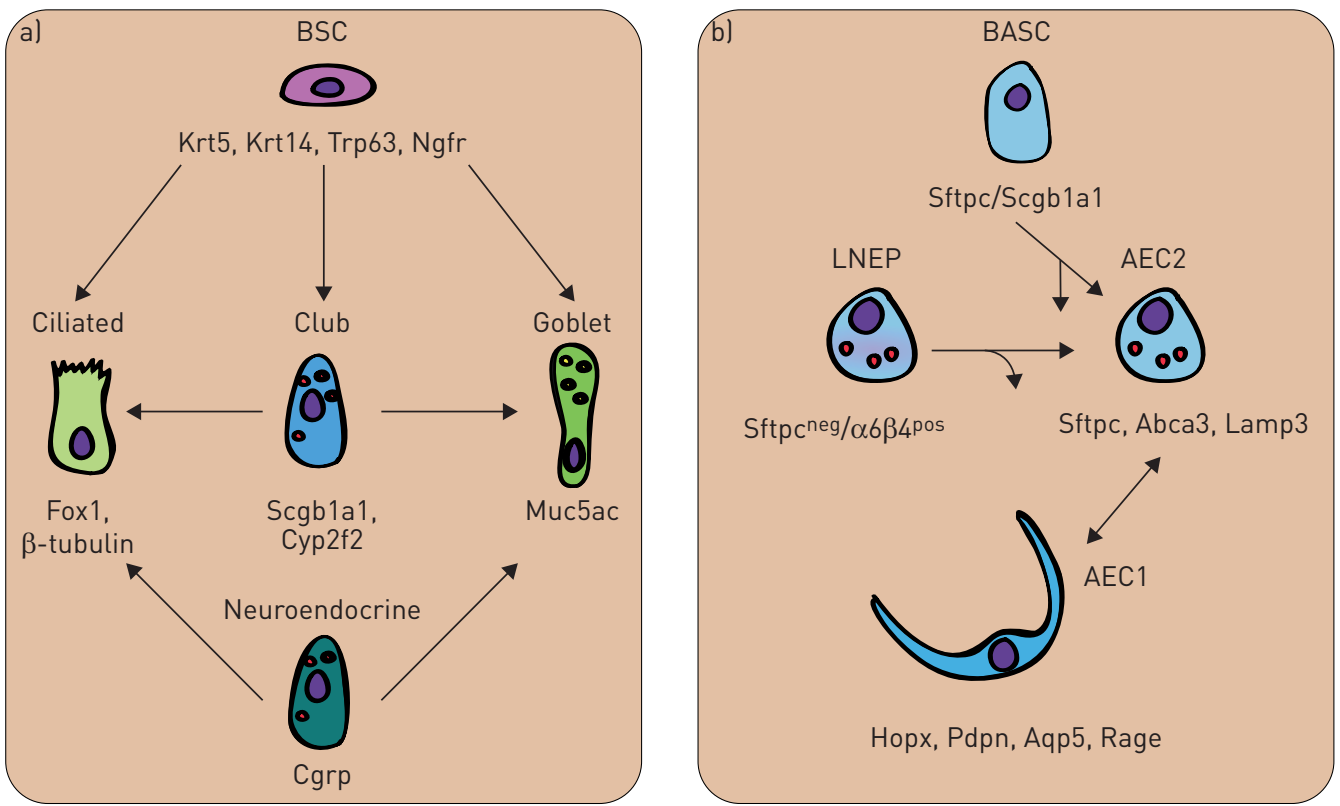

FIGURE 3 Epithelial subtypes exhibiting stem cell properties during development and repair. a) Proximal progenitor cells: basal stem cells (BSCs) expressing Krt5, Krt14, Trp63 and Ngfr can give rise to club (Scgb1a1 ${ }^{\text {pos }}$, Cyp2f2 ${ }^{\text {pos }}$ ), ciliated (Foxj1 ${ }^{\text {pos }}$, b-tubulin ${ }^{\text {pos}}$ ) and goblet (Muc5ac ${ }^{\text {pos}}$ ) cells. Neuroendocrine cells (Cgrppos) and club cells can generate both goblet and ciliated cells. b) Distal epithelial progenitors: bronchioalveolar stem cells (BASCs) co-expressing Sftpc and Scgb1a1 contribute to the generation of alveolar type 2 cells (AEC2) and alveolar type 1 cells (AEC1). Lineage-negative epithelial progenitors (LNEP) (Sftpc ${ }^{\text {neg/ }}$ $\alpha 6 \beta 4^{\mathrm{pos}}$ ) and AEC2 (Sftpc ${ }^{\mathrm{pos}}$ ) can also act as precursor cells able to self-renew and differentiate into AEC1 cells. Mouse cell type-specific markers are shown. 
In addition to BSCs, lineage tracing studies showed that secretory cells that express secretoglobin 1a1 (Scgb1a1), but lack (or have reduced) expression of cytochrome P450 family 2 subfamily $\mathrm{f}$ (Cyp2f2), are able to self-renew and re-epithelialise the airway epithelium after injury (figure 3a) [44, 59-61]. Recently, lineage tracing approaches revealed a key crosstalk between airway Scgbla1 ${ }^{\text {pos }}$ club secretory cells and specific mesenchymal population in the adult lung during injury repair [57]. In this study, LeE et al. [57] identified that Lgr6 $^{\text {pos }}$ mesenchymal cells specifically support club cell proliferation and differentiation via a paracrine Wnt-Fgf10 signalling network. Three independent studies demonstrated the use of stromal cells as support cell populations while generating mouse secretory club cell organoids derived either from a minor stem cell population isolated on the basis of being either $\mathrm{Cd} 45^{\text {neg }} \mathrm{Cd} 31^{\text {neg }} \mathrm{EpCAM}^{\text {high }} \operatorname{Itga} 6^{\text {pos }} \operatorname{Itgb} 4^{\text {pos }} \mathrm{Cd} 24^{\text {low }}$ or $\operatorname{Scgb} 1 \mathrm{a} 1^{\text {pos }}$ cells $[20,29$, 57]. Furthermore, neuroendocrine cells are also able to proliferate and generate secretory and ciliated cells after naphthalene-induced loss of secretory cells (figure 3a). However, depletion of neuroendocrine cells following injury does not impede regeneration of secretory cells, suggesting that their contribution maybe limited and/or compensated by other progenitors [62].

Recently, human tracheospheres were used to screen molecules that affect the differentiation ratio of ciliated versus secretory cells from basal cells $[53,55,56]$. This work led to the identification of a pivotal role for interleukin (IL)-13 in promoting mucus cell production and Notch signalling in inhibiting the proportion of secretory relative to ciliated cells, consistent with previous observations [17, 42, 43, 63-69]. In contrast, a similar assay reported the inhibitory effect of BMP signalling during basal cell differentiation [55]. Together, these studies highlight new potential therapeutic targets for conditions such as chronic asthma in which the epithelial balance is disrupted.

Another respiratory disease that leads to chronic bacterial infection and secretion of pro-inflammatory cytokines is cystic fibrosis (CF). hiPSCs derived from CF patients were used to evaluate the function of the CF transmembrane conductance regulator protein in airway epithelial cells and rescue the CF phenotype following treatment with small compounds $[27,39,70,71]$ or using zinc-finger nuclease-mediated gene editing approach $[27,71]$. This is another promising in vitro system that facilitates lung-specific disease modelling and drug screening.

\section{Modelling alveolar stem cells and stromal niche interaction}

Stem/progenitors also exist in the alveolus, the basic respiratory unit composed of AEC1 and AEC2 cells, capillary endothelial cells and associated distinct mesenchymal lineages. The bronchioalveolar duct junction, between the bronchioles and the alveolar sacs, consists of a small number of putative bronchioalveolar stem cells (BASCs) marked by Scgbla1 and surfactant protein C (Sftpc) co-expression, which are able to give rise to secretory and alveolar epithelial cells in vivo (figure $3 b)[17,72,73]$. Notably, a cooperative interaction between Gata6 and Wnt signalling is essential for the expansion of BASCs after naphthalene-induced airway injury [74], whereas endothelial-derived Bmp4-Nfatc1-Tsp1 signalling is crucial for BASC differentiation during lung regeneration [18]. In addition to BASCs, Sftpc ${ }^{\text {neg }} / \alpha 6 \beta 4^{\text {pos }}$ progenitors (called lineage-negative epithelial progenitors) also display regenerative potential (figure $3 \mathrm{~b}$ ) [75]. Although their regenerative capabilities have been reported to be mostly towards the alveolar lineage, it remains to be tested whether these cells can also contribute to proximal airway lineages. Finally, cell lineage tracing evidence shows that $\mathrm{Sftpc}^{\text {pos }} \mathrm{AEC} 2$ act as progenitor cells able to self-renew during homeostasis in vivo and differentiate into AEC1 cells in vitro when grown as organoids (alveolospheres) in combination with stromal cells (figure $3 \mathrm{~b}$ ) [19, 57, 76-78]. These 3D structures are composed of AEC1 in the lumen side and AEC2 cells on the outside. Of particular interest, a balance of IL-6/Stat3, Bmp and Fgf signalling proved important in AEC2 self-renewal and AEC1 differentiation [78]. Moreover, in an in vivo (kidney capsule) transplantation assay, isolated $\mathrm{Sftpc} \mathrm{c}^{\text {neg }} / \alpha 6 \beta 4^{\text {pos }}$ cells gave rise to Scgbla1 ${ }^{\text {pos }}$ club cells and Sftpc $^{\text {pos }}$ AEC2 cells [75]. In contrast, AEC1 cells, which are thought to be terminally differentiated cells in the postnatal lung, can proliferate and transdifferentiate into AEC2 after partial pneumonectomy (figure 3b) [77]. It is unclear so far how heterogeneous the AEC1 population is and whether it displays enhanced stem cell characteristics. More recently, two AEC1 cell subtypes marked by Hopx ${ }^{\text {neg }} \operatorname{Igfbp}^{\text {pos }}$ and $\mathrm{Hopx}^{\text {pos }}$ Igfbp $2^{\text {neg }}$ have been identified in the postnatal lung exhibiting distinct cell fates during alveolar regeneration following pneumonectomy [79].

In addition to alveolar stem cells, different types of resident stromal cells (which include mesenchymal and endothelial cells) are required for both homeostasis and regeneration after injury. The identification of these populations is facilitated by co-culture with epithelial lung progenitors in organoid settings. The characterisation of the cellular and molecular features of these stromal cells during repair and/or regeneration in vivo are so far elusive. It is unclear for example if these interactions are solely based on growth factors secreted by the stromal cells and/or on direct cell-to-cell contact which may or may not involve extracellular matrix components. In the alveolar niche of the adult murine distal lungs, several 
mesenchymal lineages expressing Fgf10, Gli1, or Axin2 function as multipotent cells able to self-renew and give rise to smooth muscle and fibroblast-like cells [80-83]. Notably, mouse fibroblasts have been shown to support the growth of distal epithelial stem/progenitor cells, emphasising the importance of epithelialmesenchymal interactions in close proximity [19, 29, 84]. In this regard, the Kim [57] and Morrisey [78] groups recently investigated the effect of distinct mesenchymal cell subtypes in promoting Sftpc ${ }^{\text {pos }}$ AEC2 cell self-renewal and differentiation in AEC1 cells. Using single-cell RNA sequencing, lineage tracing and organoid cultures have identified mesenchymal lineages with distinct spatial distribution and unique niche regulatory function $[57,78]$. For example, Lgr5 ${ }^{\text {pos }}$ alveolar mesenchymal cells are able to induce alveolar lineage differentiation of epithelial progenitors $\left(\mathrm{Scgbla1}{ }^{\text {pos }}\right.$ or $\mathrm{Sftpc}^{\text {pos }}$ cells) by secreting Wnt ligands [57]. In addition to Lgr $^{\text {pos }}$ population, the Axin2 ${ }^{\text {pos}} /$ Pdgfra $^{\text {pos }}$ alveolar mesenchymal lineage is capable of promoting AEC2 self-renewal via Il-6 and Fgf7 signalling [78]. An additional challenge lies in determining whether these alveolar mesenchymal populations are two distinct mesenchymal cell subtypes.

Given the close proximity of the pulmonary capillary endothelial cells (PCECs) to the alveolar epithelial cells in vivo, endothelial-derived membrane-bound and soluble angiocrine factors have significant input for alveolar stem cell differentiation [18, 85-87]. AEC1 and AEC2 cells are lined with PCECs that are positive for Cd31, Cd34, Fgfr-1, Vegfr-1, Vegfr-2, negative for Cd45 [85]. In vivo studies using unilateral pneumonectomy as a model for neo-alveolarisation demonstrated that platelet-derived Sdf-1 leads to the upregulation of Mmp-14 in PCECs, which in turn triggers alveolar stem cell proliferation by unmasking the cryptic Egf-like fragment in Hb-Egf and the $\gamma 2$ chain of laminin-5 [85, 87]. Mouse organotypic study of epithelial stem cells (EpCAM ${ }^{\text {pos }} \mathrm{Sca}^{\text {pos }} \mathrm{Cd} 31^{\text {neg }} \mathrm{Cd} 45^{\text {neg }}$ ) that were co-cultured with primary mouse lung endothelial cells (LuMECs) support a model in which angiocrine signals regulate alveolar stem cell proliferation and differentiation into AEC1 and AEC2 cells [18]. LuMECs have been shown to produce Tsp-1, which fosters lung stem cell differentiation to the alveolar lineage.

For disease modelling, CHEN et al. [28] established the first proof-of-principle hPSC-based modelling of Hermansky-Pudlak syndrome (HPS) which leads to pulmonary fibrosis with abnormalities in AEC2 and surfactant proteins secretion in vivo. To study the effect of the gene HSP1 gene in human development in vitro, they generated lung bud organoid from RUES2 hPSCs with CRISPR-Cas9 gene editing induced deletion of HPS1 [28]. Notably, this study provided consistent evidence [88, 89] that mutant epithelial cells are responsible for the accumulation of mesenchymal cells and increased ECM deposition [28].

\section{Development of human organs-on-chips}

Recent advances in the development of microengineered cell culture systems provide new opportunities to present cultured living cells with physiologically relevant biochemical and mechanical cues with high spatiotemporal precision (figure 4). These biomimetic in vitro models collective known as organs-on-chips are constructed in microfabricated devices that often consist of two or more layers of cell culture chambers made out of optically transparent and biocompatible polymeric materials such as poly(dimethylsiloxane) (PDMS) using soft lithography-based replica moulding techniques. This widely used multilayered design provides compartmentalised environments for co-culture of multiple cell types under dynamic flow conditions to engineer various kinds of tissue-tissue interfaces and 3D multicellular structures that mimic the functional units of living human organs (figures 2 and 4a) [90]. In vitro approaches based on organ-on-a-chip technology have proven instrumental in modelling the complexity and dynamic nature of the respiratory system (supplementary figure S1). Here we discuss representative examples of such systems to demonstrate the proven utility and potential of lung-on-a-chip technology.

\section{Early demonstrations of human lung-on-a-chip}

Lung-on-a-chip studies focused on demonstrating the possibility of using multilayered microfluidic devices to enable the formation of differentiated airway epithelium with morphological and secretory phenotypes matching those found in vivo [91-96]. For example, a human small airway-on-a-chip model developed by Takayama and co-workers [94] permitted long-term ( $>3$ weeks) air-liquid interface culture of primary human small airway epithelial cells to produce a differentiated airway epithelium with structural integrity and barrier function. More importantly, this culture system was integrated with a microfluidic plug generator capable of creating microscopic liquid plugs that were actuated to propagate over the epithelial surface and rupture to mimic closure and reopening of small airways due to mucus plugs in diseased lungs. This dynamic airway-on-a-chip model showed that plug propagation and rupture can generate abnormally large mechanical forces and harm the epithelium in a dose-dependent manner [94]. Furthermore, the development of bifurcating microchannel network enabled to investigate the dynamics of plug rupture events in independent subnetworks, which then evolve independently of one another as demonstrated by the work from Baroud and co-workers [97-99]. Combined with the engineering novelty 
a)
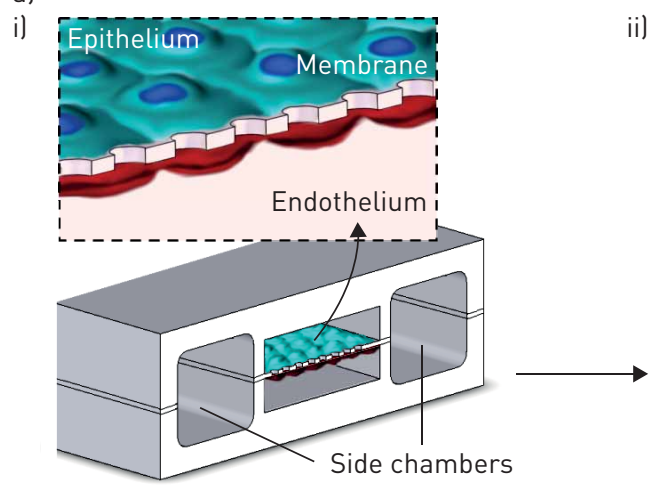

ii) $\Gamma$

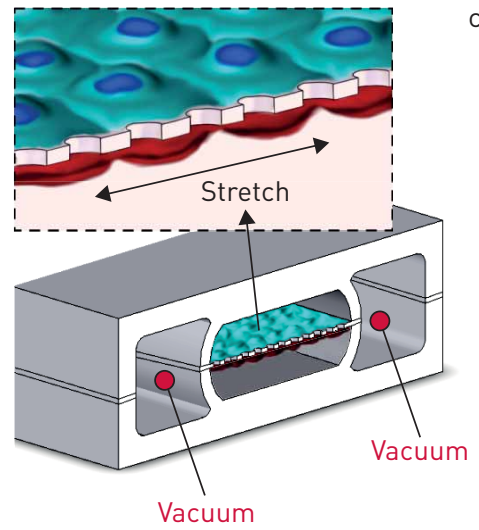

b)

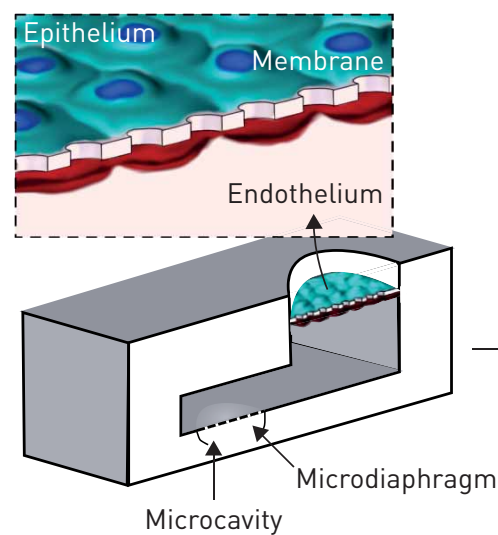

c)
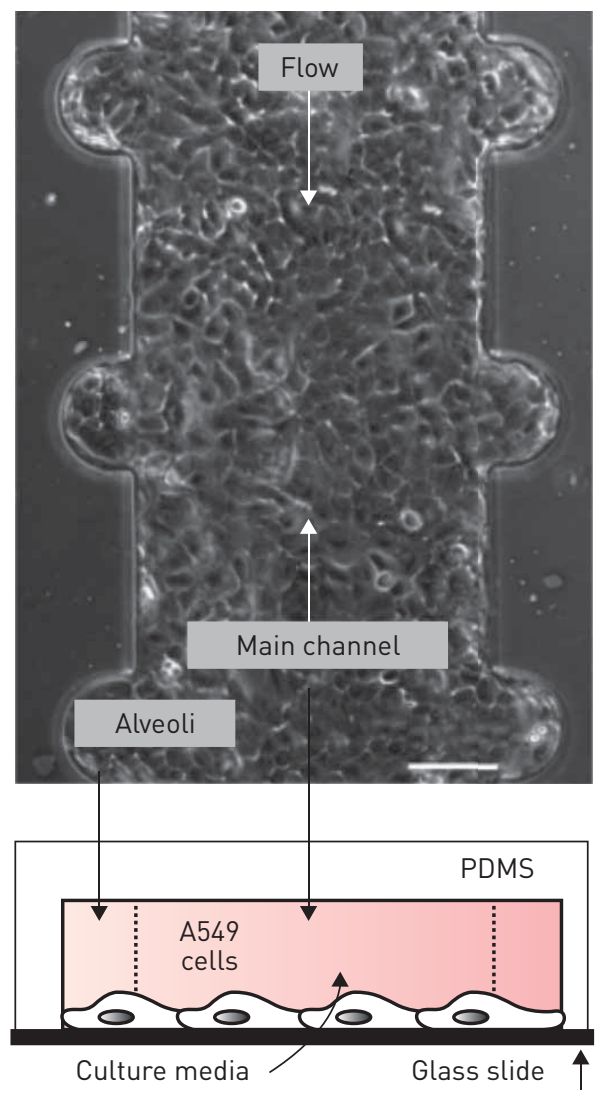

FIGURE 4 Microengineered models of the human lung-on-a-chip. a) i) Schematic representation of cross-section through the lung-on-a-chip model containing two poly(dimethylsiloxane) (PDMS) compartments separated by a thin porous membrane and surrounded by two vacuum chambers. The top "air" compartment is lined by human epithelial cells; the bottom "liquid" compartment is cultured with human endothelial cells; ii) vacuum can be applied to cyclically stretch the microengineered epithelial-endothelial barrier and to mimic physiological breathing motions. b) i) Schematic representation of lung-on-a-chip model containing two PDMS compartments separated by a stretchable membrane to form the alveolar barrier. The lower compartment is filled with culture medium and a PDMS membrane (microdiaphragm) at the bottom of the compartment is surrounded by a cavity; ii) the alveolar barrier is stretched downwards a negative pressure applied in the cavity. c) Alveolated microfluidic channel lined with regularly positioned cylindrical cavities replicating the in vivo acinar morphology. Scale bar=100 $\mu \mathrm{m}$. Reproduced and modified from [106] with permission from the publisher.

of the system, this new physiological insight provided strong evidence for the advantages and potential of lung-on-a-chip systems as a novel research platform.

This demonstration was followed by another major study which showed for the first time the feasibility of reconstituting complex and integrated organ-level physiological responses of the lung in a microengineered device [100]. This system was designed to mimic the mechanically active alveolar-capillary unit of the living human lung. The device was created in a multicompartment microfluidic system in which human alveolar epithelial cells were cultured in close apposition to primary human pulmonary microvascular endothelial cells on a thin porous elastomeric membrane to form a barrier tissue reminiscent of the alveolar-capillary interface in vivo. Inspired by the mechanism of breathing in the lung, the authors also devised a novel mechanical actuation scheme based on controlled application of vacuum to cyclically stretch the microengineered alveolar-capillary barrier and to mimic physiological breathing motions (figure 4). Importantly, this study demonstrated the novel capability of the human breathing lung-on-a-chip model to mimic complex integrated organ-level responses. For example, when the alveolar epithelium was exposed to pathogenic bacteria in this microdevice, the epithelial cells released inflammatory cytokines and activated the microvascular endothelial cells on the opposite of the membrane, inducing them to express high levels of adhesion molecules such as intercellular adhesion molecule-1. Primary human neutrophils circulating in the lower capillary channel recognised these activated endothelial cells and established firm adhesion to the endothelium, after which the adhered neutrophils underwent transmigration across the alveolar-capillary barrier. 
In addition, the ability of this model to recapitulate dynamic mechanical activity of the lung led to the discovery of previously unexplained adverse effects of physiological breathing-induced mechanical forces on inflammatory and injury responses. For example, in nanotoxicology studies using silica nanoparticles that simulated air pollutants, cyclic breathing motions in the lung-on-a-chip system substantially increased endothelial expression of pro-inflammatory adhesion molecules and intracellular production of reactive oxygen species, suggesting the promotive effects of physiological breathing on acute toxic responses to environmental particulates.

This advanced lung-on-a-chip platform was used in the follow-on study to test the feasibility of engineering specialised models of lung diseases. To this end, the authors created a microengineered model that simulated the development and progression of pulmonary oedema induced by dose-limiting toxicity of a chemotherapeutic drug, IL-2 [101]. Furthermore, this mechanically active model capable of mimicking physiological breathing motions revealed the promotive effects of breathing-generated mechanical forces on IL-2-induced tissue injury, which was effectively inhibited by potential drug candidates such as angiopoietin-1 and a newly developed transient receptor potential vallinoid 4 ion channel blocker. In another study, Long et al. [102] used mathematical modelling to optimise and control gas concentrations within the gas and liquid sides of the alveolus-capillary interface, thus enhancing the performance of the system for drug and toxicity studies. Another "breathing" lung-on-a-chip system was developed by Guenat and co-workers [103] to mimic the alveolar system of the lung (figure 4b). This model consists of a PDMS cell culture well and an underlying chamber separated by a thin PDMS membrane with microscopic pores. In this multilayered device, primary human pulmonary alveolar epithelial cells and endothelial cells were cultured on the opposite sides of the membrane to form a bilayer tissue structure reminiscent of the alveolar-capillary interface. Using mechanical actuation inspired by the mechanism of respiration in the lung, this system made it possible to simulate breathing-induced 3D deformation of the alveolar air sacs. Specifically, the lower chamber of the device was equipped with a membrane-based actuation system where negative pressure was applied to induce the deflection of a diaphragm membrane, which led to downward deformation of the engineered alveolar-capillary barrier (figure $4 \mathrm{~b}$ ). Importantly, this study demonstrated that breathing-induced mechanical strain influences barrier permeability, the metabolic activity and the cytokine secretion (IL-8) of primary human pulmonary alveolar epithelial cells obtained from patients [103].

Recently, Davies and co-workers [104] developed a 3D in vitro model by co-culturing human epithelial cell line and human umbilical vein endothelial cells (HUVECs) at the air-liquid interface. This system was applied to dissect the temporal crosstalk between epithelial and endothelial barriers in response to viral infections. While this work successfully recapitulated human pulmonary viral infection in vitro, additional progress is needed to evaluate the co-culture model using fully differentiated primary airway/bronchial epithelial and microvascular endothelial cells. Another example of microengineered human lung models can be found in the work of SELLGREN et al. [105], which developed vertically stacked and individually accessible 3D culture compartments for culture of primary human airway cells. This platform allows for co-culture of trachea-bronchial epithelial cells, lung fibroblast and microvascular endothelial cells at the upper, middle and lower compartments, respectively. The authors observed well-differentiated epithelial cells that exhibited physiological tissue-specific phenotypes including barrier function.

\section{Development of alveolated microchannels}

Inspired by the innate acinar morphology of the alveoli, researchers have explored the possibility of using single or bifurcating microchannel networks combined with cylindrical alveolar cavities (figure 4c) to study airflow mixing in the pulmonary acinus and to predict the fate of inhaled aerosols in the distal airway [106-109]. For example, Snitzman and co-workers [106] created a model that mimics the alveolar morphology, flow patterns and other important physiological characteristics aspects of fetal lungs developing in utero. Furthermore, this platform enabled time-resolved imaging of inhaled particle flight trajectories and ensuing respiratory acinar flows [109]. This study demonstrated the complexity of acinar particle dynamics that result from the coupling between intrinsic transport mechanisms (gravity and diffusion) and the complex local alveolar airflow patterns.

\section{Recent advances in lung-on-a-chip technology}

Early investigations described earlier exemplify an array of opportunities enabled by lung-on-a-chip technology for prediction and mechanistic investigation of complex physiological responses of the human lung. Building upon the success of these studies, researchers have recently reported several lung-on-a-chip systems that model various aspects of lung physiology and pathophysiology. Here we describe representative examples of these models. 


\section{Small airway-on-a-chip}

Lung-on-a-chip model systems enable unprecedented study of the synergistic crosstalk between multiple cell types that drive pathological processes in the human lung, as demonstrated by the work of BENAM et al. [110]. In this study, the research team engineered a human "small airway-on-a-chip" to study the influence of epithelial-endothelial crosstalk on inflammatory cytokine production and neutrophil recruitment in the small airways of patients with diseases such as asthma and chronic obstructive pulmonary disease (COPD). The upper microfluidic channel was seeded with human small airway epithelial cells later cultured at the air-liquid interface to facilitate mucociliatory differentiation, while the lower microfluidic channel was seeded with lung microvascular endothelial cells and perfused to create a physiological milieu capable of modelling organ-level inflammatory disease processes. First, to model goblet cell hyperplasia, cytokine hypersecretion and mucociliatory dysfunction seen in the small airways of asthmatics, supraphysiological concentrations of IL-13 were added to the small airway-on-a-chip via the medium perfused in the microvascular channel. The use of COPD patient-derived cells in this device recapitulated key disease features including selective cytokine secretion, increased neutrophil recruitment and exacerbation-like increases in these responses upon introduction of viral and bacterial pathogens. In addition, this system was treated with IL-13 to create a disease model that recapitulated certain features of asthma, which was used to demonstrate quantitative measurement of clinically relevant outputs upon treatment with anti-inflammatory compounds, including reduced cytokine production, decreased goblet cell hyperplasia and recovery of normal ciliary beat frequency.

\section{Organotypic lung-on-a-chip model for the study of host-pathogen interactions}

Lung-on-a-chip technology provides new opportunities to model pathophysiologically relevant hostpathogen interactions by allowing for co-culture of lung cells with living bacteria, virus or fungi in a controlled manner. A recent example of modelling host-pathogen interactions in the lungs can be found in the work of BARKAL et al. [111] which established a microengineered model of the human bronchiole used to study the early stages of Aspergillus fumigatus infection in human lungs.

A central lumen was seeded with small airway epithelial cells to form the bronchiole, and microvascular endothelial cells were added to the two remaining lumens to form perfusable vessels. Additionally, fibroblasts were embedded in the surrounding collagen hydrogel to model the stromal tissue compartment. To model the small airway response to inhaled pathogens, the central airway lumen was exposed to A. fumigatus, a common fungal pathogen responsible for respiratory infections. Following exposure, the fungus traversed the epithelial barrier and formed typical fungal hyphae structures in the surrounding collagen hydrogel. To model the first line of host defence, human neutrophils were added to the perfused microvascular lumens where some cells were shown to enter the surrounding hydrogel and migrate toward fungal hyphae structures.

Leveraging the inherent capacity of microengineered models to physically separate and fluidically connect different types of cells or microbes, the authors created a closed device containing separate chambers of A. fumigatus and the bacterium Pseudomonas aeruginosa along with three human bronchiole modules to facilitate volatile communication between the pathogens and the airway epithelial cells via open airway lumens. While this work successfully recapitulated human pulmonary fungal infection in vitro, much additional progress is needed to realise clinically relevant models of pulmonary infection that will help inform a better understanding of how to boost the early host response to pathogen exposure.

\section{Alveolus-on-a-chip model of intravascular thrombosis}

Although pulmonary thrombosis is a significant cause of patient mortality, there exist no traditional in vitro models of microvascular thrombi formation. Additionally, animal models of pulmonary microvascular thrombosis fail to capture the complex haemodynamic behaviour of human lungs. To address these limitations, JAIN et al. [112] presented a microfluidic alveolus-on-a-chip model that recapitulated the organ-level pathophysiological effects of pulmonary thrombosis.

Primary human alveolar epithelial cells were cultured at the air-liquid interface along the membrane in the upper compartment, and HUVECs lined all four sides of the lower compartment to form a continuous lumen. This platform enabled the perfusion of whole blood through the lower vascular compartment without thrombus formation nor platelet adhesion in healthy engineered microvessels. Following stimulation with tumour necrosis factor (TNF)- $\alpha$; however, the system exhibited thrombus formation and rapid platelet recruitment. In addition, the aggregating platelets in this condition formed a teardrop shape that mirrored in vivo observations, which is absent in ECM-coated microfluidic devices traditionally used to study thrombus formation. Additionally, the system was used to study lipopolysaccharide (LPS) endotoxin-induced thrombus formation, revealing that tissue-tissue interactions between the alveolar epithelium and vascular endothelium mediate the prothrombotic response through an epithelium-generated 
cytokine cascade. Lastly, the application of the alveolus model in preclinical drug development was shown by demonstrating the cytoprotective and antithrombotic activity of permodulin-2, a potent inhibitor of inflammation-mediating protease activated receptor-1, in the presence of LPS.

\section{Challenges and future directions}

Organoid and lung-on-a-chip technologies are able to recapitulate several basic functions of the lung, but a number of obstacles need to be tackled as the field develops into preclinical applications. Despite the obvious benefit of organoids in recapitulating cellular heterogeneity and physiologically relevant complexity, it is still unclear how useful organoids will be for studying dynamic interactions under physiological vascular shear. In this particular case, the field will probably overcome this limitation through synergistic engineering between organoid and lung-on-a-chip approaches (organoid-on-a-chip) [113]. Thus, it is important to accelerate the combined engineering and stem cell expertise among the scientific community. Unlike organoids, the lung-on-a-chip system is able to reproduce key dynamic processes, including immune-endothelial cell interactions under blood flow and inhalation/exhalation-associated airflow. Taking it a step further, this technology can be used to reconstitute disease-specific biological responses to smoke or fine particles and identify molecular signatures that can serve as potential therapeutic targets or diagnostic biomarkers [114]. Given the complex composition of the fluid in the organoid lumen and its challenging accessibility, it is currently unclear whether the organoid system can be adapted to similar air stimuli. Furthermore, the primary concern regarding the use of hPSC-derived organoids is that current differentiation protocols often mimic the second trimester of fetal development. This concern is a major drawback, especially when organoids are used for modelling adult diseases and challenging clinical applications such as drug development, screening and personalised medicine. As mentioned above, mechanical forces of the ECM, flow and inputs from the surrounding mesenchymal-endothelial cells might play a key role in promoting such maturation events. The development of organoid-on-a-chip could overcome some of these limitations and generate fully differentiated human epithelial cells. In line with this idea, the use of hPSCs as a source of cells for lung-on-a-chip system has a number of advantages. For example, hPSCs provide a potentially unlimited source of different cell types which can be a cost-effective and more robust source than primary cells. Furthermore, given the major challenges in obtaining human lung tissues from patients and healthy individuals, the use of hPSCs combined with gene editing technologies (e.g. CRISPR) to generate disease-causing mutations can eliminate the need for invasive procedures, such as biopsy and bronchoscopy brushing. One of the most difficult obstacles to overcome when using organotypic cultures is the challenge of generating organoids of a uniform size and controlling cell ratios during self-organisation. In contrast, the lung-on-a-chip approach where cells are cultured in a defined orientation and in a uniform manner represents a more reproducible cell culture system. This organoid variation makes it hard to incorporate microsensors for critical control and functional parameters (e.g. barrier integrity, cell migration, flow, oxygen, glucose, fluid pressure), which has been proved to be enormously useful in organ-on-a-chip systems [115-117]. Despite the remarkable progress in lung-on-a-chip technology, another challenge to overcome is that the ECM-coated PDMS membranes that mimic tissue interface may have different transport and structural properties from those seen in vivo.

In summary, both organoid and lung-on-a-chip approaches offer a wealth of opportunities, not only to investigate mechanisms involved in lung development, regeneration and disease, but also to screen for drugs which may eventually lead to more efficient treatments of debilitating lung diseases.

Acknowledgements: We thank Elie El Agha (Justus-Liebig University Giessen, Excellence Cluster Cardio-Pulmonary System, Universities of Giessen and Marburg Lung Centre, German Center for Lung Research, Giessen, Germany) for kindly providing unpublished data shown in supplementary figure S1.

Conflict of interest: K. Gkatzis has nothing to declare. S. Taghizadeh has nothing to declare. D. Huh has US Patent 86478616 B2 licensed, US patent 7704728 licensed, and patents US 62/544,429, US $62 / 348,055$ and US $62 / 348,036$ pending. D. Huh owns shares in Emulate, Inc. and consults for the company. D.Y.R. Stainier has nothing to declare. S. Bellusci has nothing to declare.

Support statement: S. Bellusci is supported by the German Research Foundation (DFG; BE4443/4-1, BE4443/6-1, KFO 309 and CRC1213), Landes-Offensive zur Entwicklung Wissenschaftlich-okonomischer Exzellenz (LOEWE), University Hospital of Giessen and Marburg, Universities of Giessen and Marburg Lung Center, the German Centre for Lung Research (DZL), COST (BM1201) and National Institutes of Health National Heart, Lung, and Blood Institute (1R01HL086322-01A2 and HL107307). Work in the Stainier lab is supported by funds from the Max Planck Society, European Union (European Research Council), Deutsche Forschungsgemeinschaft and Leducq Foundation. Due to space limitations, we could not cover all relevant work done in this field.

\section{References}

1 Hogan BLM, Barkauskas CE, Chapman HA, et al. Repair and regeneration of the respiratory system: complexity, plasticity, and mechanisms of lung stem cell function. Cell Stem Cell 2014; 15: 123-138. 
Metzger RJ, Klein OD, Martin GR, et al. The branching programme of mouse lung development. Nature 2008; 453: 745-750. bifurcation during branching morphogenesis of the mammalian lung. Dev Cell 2015; 34: 719-726.

Herriges M, Morrisey EE. Lung

Development 2014; 141: 502-513.

Desai TJ, Brownfield DG, Krasnow MA. Alveolar progenitor and stem cells in lung development, renewal and cancer. Nature 2014; 507: 190-194.

disease. Biochem Biophys Res Commun 2016; 473: 675-682.

9 Clevers H. Modeling development and disease with organoids. Cell 2016; 165: 1586-1597.

10 Mondrinos MJ, Jones PL, Finck CM, et al. Engineering de novo assembly of fetal pulmonary organoids. Tissue Eng Part A 2014; 20: 2892-2907.

11 Mondrinos MJ, Koutzaki S, Lelkes PI, et al. A tissue-engineered model of fetal distal lung tissue. Am J Physiol Lung Cell Mol Physiol 2007; 293: L639-L650.

12 Zhang S, Zhou X, Chen T, et al. Single primary fetal lung cells generate alveolar structures in vitro. In Vitro Cell Dev Biol Anim 2014; 50: 87-93.

13 Nichane M, Javed A, Sivakamasundari V, et al. Isolation and 3D expansion of multipotent Sox $9^{+}$mouse lung progenitors. Nat Methods 2017; 14: 1205-1212.

14 Nikolić MZ, Caritg O, Jeng Q, et al. Human embryonic lung epithelial tips are multipotent progenitors that can be expanded in vitro as long-term self-renewing organoids. Elife 2017; 6: 4139.

15 Miller AJ, Hill DR, Nagy MS, et al. In vitro induction and in vivo engraftment of lung bud tip progenitor cells derived from human pluripotent stem cells. Stem Cell Reports 2018; 10: 101-119.

16 Rock JR, Onaitis MW, Rawlins EL, et al. Basal cells as stem cells of the mouse trachea and human airway epithelium. Proc Natl Acad Sci USA 2009; 106: 12771-12775.

17 Rock JR, Gao X, Xue Y, et al. Notch-dependent differentiation of adult airway basal stem cells. Cell Stem Cell 2011; 8: 639-648.

18 Lee J-H, Bhang DH, Beede A, et al. Lung stem cell differentiation in mice directed by endothelial cells via a BMP4-NFATc1-thrombospondin-1 axis. Cell 2014; 156: 440-455.

19 Barkauskas CE, Cronce MJ, Rackley CR, et al. Type 2 alveolar cells are stem cells in adult lung. J Clin Invest 2013; 123: 3025-3036.

20 McQualter JL, Yuen K, Williams B, et al. Evidence of an epithelial stem/progenitor cell hierarchy in the adult mouse lung. Proc Natl Acad Sci USA 2010; 107: 1414-1419.

21 Zacharias WJ, Frank DB, Zepp JA, et al. Regeneration of the lung alveolus by an evolutionarily conserved epithelial progenitor. Nature 2018; 555: 251-255.

22 Fox E, Shojaie S, Wang J, et al. Three-dimensional culture and FGF signaling drive differentiation of murine pluripotent cells to distal lung epithelial cells. Stem Cells Dev 2015; 24: 21-35.

23 Gotoh S, Ito I, Nagasaki T, et al. Generation of alveolar epithelial spheroids via isolated progenitor cells from human pluripotent stem cells. Stem Cell Reports 2014; 3: 394-403.

24 Konishi S, Gotoh S, Tateishi K, et al. Directed induction of functional multiciliated cells in proximal airway epithelial spheroids from human pluripotent stem cells. Stem Cell Reports 2016; 6: 18-25.

25 Dye BR, Hill DR, Ferguson MAH, et al. In vitro generation of human pluripotent stem cell derived lung organoids. Elife 2015; 4: 1999.

26 Yamamoto Y, Gotoh S, Korogi Y, et al. Long-term expansion of alveolar stem cells derived from human iPS cells in organoids. Nat Methods 2017; 14: 1097-1106.

27 McCauley KB, Hawkins F, Serra M, et al. Efficient derivation of functional human airway epithelium from pluripotent stem cells via temporal regulation of Wnt signaling. Cell Stem Cell 2017; 20: 844-857.

28 Chen Y-W, Huang SX, de Carvalho ALRT, et al. A three-dimensional model of human lung development and disease from pluripotent stem cells. Nat Cell Biol 2017; 19: 542-549.

29 Chen H, Matsumoto K, Brockway BL, et al. Airway epithelial progenitors are region specific and show differential responses to bleomycin-induced lung injury. Stem Cells 2012; 30: 1948-1960.

30 Wilkinson DC, Alva-Ornelas JA, Sucre JMS, et al. Development of a three-dimensional bioengineering technology to generate lung tissue for personalized disease modeling. Stem Cells Transl Med 2017; 6: 622-633.

31 Zorn AM, Wells JM. Vertebrate endoderm development and organ formation. Annu Rev Cell Dev Biol 2009; 25 221-251.

32 Wong AP, Bear CE, Chin S, et al. Directed differentiation of human pluripotent stem cells into mature airway epithelia expressing functional CFTR protein. Nat Biotechnol 2012; 30: 876-882.

33 Mou H, Zhao R, Sherwood R, et al. Generation of multipotent lung and airway progenitors from mouse ESCs and patient-specific cystic fibrosis iPSCs. Cell Stem Cell 2012; 10: 385-397.

34 Huang SXL, Islam MN, O’Neill J, et al. Efficient generation of lung and airway epithelial cells from human pluripotent stem cells. Nat Biotechnol 2014; 32: 84-91.

35 Firth AL, Dargitz CT, Qualls SJ, et al. Generation of multiciliated cells in functional airway epithelia from human induced pluripotent stem cells. Proc Natl Acad Sci USA 2014; 111: E1723-E1730.

36 Green MD, Chen A, Nostro MC, et al. Generation of anterior foregut endoderm from human embryonic and induced pluripotent stem cells. Nat Biotechnol 2011; 29: 267-272.

37 Longmire TA, Ikonomou L, Hawkins F, et al. Efficient derivation of purified lung and thyroid progenitors from embryonic stem cells. Cell Stem Cell 2012; 10: 398-411.

38 Ghaedi M, Calle EA, Mendez JJ, et al. Human iPS cell-derived alveolar epithelium repopulates lung extracellular matrix. J Clin Invest 2013; 123: 4950-4962.

39 Hannan NRF, Sampaziotis F, Segeritz C-P, et al. Generation of distal airway epithelium from multipotent human foregut stem cells. Stem Cells Dev 2015; 24: 1680-1690. 
Danopoulos S, Alonso I, Thornton M, et al. Human lung branching morphogenesis is orchestrated by the spatiotemporal distribution of ACTA2, SOX2 and SOX9. Am J Physiol Lung Cell Mol Physiol 2018; 314: L144-L149.

Morimoto M, Nishinakamura R, Saga Y, et al. Different assemblies of Notch receptors coordinate the distribution of the major bronchial Clara, ciliated and neuroendocrine cells. Development 2012; 139: 4365-4373.

Guseh JS, Bores SA, Stanger BZ, et al. Notch signaling promotes airway mucous metaplasia and inhibits alveolar development. Development 2009; 136: 1751-1759.

Tsao P-N, Vasconcelos M, Izvolsky KI, et al. Notch signaling controls the balance of ciliated and secretory cell fates in developing airways. Development 2009; 136: 2297-2307.

Rawlins EL, Clark CP, Xue Y, et al. The Id $2^{+}$distal tip lung epithelium contains individual multipotent embryonic progenitor cells. Development 2009; 136: 3741-3745.

McIntyre BA, Alev C, Mechael R, et al. Expansive generation of functional airway epithelium from human embryonic stem cells. Stem Cells Transl Med 2013; 3: 7-17.

Huang SXL, Green MD, de Carvalho AT, et al. The in vitro generation of lung and airway progenitor cells from human pluripotent stem cells. Nat Protoc 2015; 10: 413-425.

Dye BR, Dedhia PH, Miller AJ, et al. A bioengineered niche promotes in vivo engraftment and maturation of pluripotent stem cell derived human lung organoids. Elife 2016; 5: 3025.

Hawkins F, Kramer P, Jacob A, et al. Prospective isolation of NKX2-1-expressing human lung progenitors derived from pluripotent stem cells. J Clin Invest 2017; 127: 2277-2294.

Ghaedi M, Mendez JJ, Bove PF, et al. Alveolar epithelial differentiation of human induced pluripotent stem cells in a rotating bioreactor. Biomaterials 2014; 35: 699-710.

Jacob A, Morley M, Hawkins F, et al. Differentiation of human pluripotent stem cells into functional lung alveolar epithelial cells. Cell Stem Cell 2017; 21: 472-488.

Chen Y, Feng J, Zhao S, et al. Long-term engraftment promotes differentiation of alveolar epithelial cells from human embryonic stem cells derived lung organoids. Stem Cells Dev 2018; 27: 1339-1349.

Butler CR, Hynds RE, Gowers KHC, et al. Rapid expansion of human epithelial stem cells suitable for airway tissue engineering. Am J Respir Crit Care Med 2016; 194: 156-168.

Danahay H, Pessotti AD, Coote J, et al. Notch2 is required for inflammatory cytokine-driven goblet cell metaplasia in the lung. Cell Rep 2015; 10: 239-252.

Gao X, Bali AS, Randell SH, et al. GRHL2 coordinates regeneration of a polarized mucociliary epithelium from basal stem cells. J Cell Biol 2015; 211: 669-682.

Tadokoro T, Gao X, Hong CC, et al. BMP signaling and cellular dynamics during regeneration of airway epithelium from basal progenitors. Development 2016; 143: 764-773.

Tadokoro T, Wang Y, Barak LS, et al. IL-6/STAT3 promotes regeneration of airway ciliated cells from basal stem cells. Proc Natl Acad Sci USA 2014; 111: E3641-E3649.

Lee J-H, Tammela T, Hofree M, et al. Anatomically and functionally distinct lung mesenchymal populations marked by Lgr5 and Lgr6. Cell 2017; 170: 1149-1163.

Hild M, Jaffe AB. Production of 3-D airway organoids from primary human airway basal cells and their use in high-throughput screening. Curr Protoc Stem Cell Biol 2016; 37: IE.9.1-IE.9.15.

Giangreco A, Reynolds SD, Stripp BR. Terminal bronchioles harbor a unique airway stem cell population that localizes to the bronchoalveolar duct junction. Am J Pathol 2002; 161: 173-182.

Tata PR, Mou H, Pardo-Saganta A, et al. Dedifferentiation of committed epithelial cells into stem cells in vivo. Nature 2013; 503: 218-223

Hong KU, Reynolds SD, Giangreco A, et al. Clara cell secretory protein-expressing cells of the airway neuroepithelial body microenvironment include a label-retaining subset and are critical for epithelial renewal after progenitor cell depletion. Am J Respir Cell Mol Biol 2001; 24: 671-681.

Song H, Yao E, Lin C, et al. Functional characterization of pulmonary neuroendocrine cells in lung development, injury, and tumorigenesis. Proc Natl Acad Sci USA 2012; 109: 17531-17536.

Dabbagh K, Takeyama K, Lee HM, et al. IL-4 induces mucin gene expression and goblet cell metaplasia in vitro and in vivo. J Immunol 1999; 162: 6233-6237.

Grünig G, Warnock M, Wakil AE, et al. Requirement for IL-13 independently of IL-4 in experimental asthma. Science 1998; 282: 2261-2263.

Munitz A, Brandt EB, Mingler M, et al. Distinct roles for IL-13 and IL-4 via IL-13 receptor $\alpha 1$ and the type II IL-4 receptor in asthma pathogenesis. Proc Natl Acad Sci USA 2008; 105: 7240-7245.

Atherton HC, Jones G, Danahay H. IL-13-induced changes in the goblet cell density of human bronchial epithelial cell cultures: MAP kinase and phosphatidylinositol 3-kinase regulation. Am J Physiol Lung Cell Mol Physiol 2003; 285: L730-L739.

Kuperman DA, Huang X, Koth LL, et al. Direct effects of interleukin-13 on epithelial cells cause airway hyperreactivity and mucus overproduction in asthma. Nat Med 2002; 8: 885-889.

Laoukili J, Perret E, Willems T, et al. IL-13 alters mucociliary differentiation and ciliary beating of human respiratory epithelial cells. J Clin Invest 2001; 108: 1817-1824.

Deblandre GA, Wettstein DA, Koyano-Nakagawa N, et al. A two-step mechanism generates the spacing pattern of the ciliated cells in the skin of Xenopus embryos. Development 1999; 126: 4715-4728.

Wong AP, Chin S, Xia S, et al. Efficient generation of functional CFTR-expressing airway epithelial cells from human pluripotent stem cells. Nat Protoc 2015; 10: 363-381.

Crane AM, Kramer P, Bui JH, et al. Targeted correction and restored function of the CFTR gene in cystic fibrosis induced pluripotent stem cells. Stem Cell Reports 2015; 4: 569-577.

Kim CFB, Jackson EL, Woolfenden AE, et al. Identification of bronchioalveolar stem cells in normal lung and lung cancer. Cell 2005; 121: 823-835.

Tropea KA, Leder E, Aslam M, et al. Bronchioalveolar stem cells increase after mesenchymal stromal cell treatment in a mouse model of bronchopulmonary dysplasia. Am J Physiol Lung Cell Mol Physiol 2012; 302: L829-L837.

Zhang Y, Goss AM, Cohen ED, et al. A Gata6-Wnt pathway required for epithelial stem cell development and airway regeneration. Nat Genet 2008; 40: 862-870.

Chapman HA, Li X, Alexander JP, et al. Integrin $\alpha 6 \beta 4$ identifies an adult distal lung epithelial population with regenerative potential in mice. J Clin Invest 2011; 121: 2855-2862. 
Evans MJ, Cabral LJ, Stephens RJ, et al. Renewal of alveolar epithelium in the rat following exposure to $\mathrm{NO}_{2}$. Am J Pathol 1973; 70: 175-198.

Jain R, Barkauskas CE, Takeda N, et al. Plasticity of $\mathrm{Hopx}^{+}$type I alveolar cells to regenerate type II cells in the lung. Nat Commun 2015; 6: 6727.

Zepp JA, Zacharias WJ, Frank DB, et al. Distinct mesenchymal lineages and niches promote epithelial self-renewal and myofibrogenesis in the lung. Cell 2017; 170: 1134-1148.

Wang Y, Tang Z, Huang H, et al. Pulmonary alveolar type I cell population consists of two distinct subtypes that differ in cell fate. Proc Natl Acad Sci USA 2018; 115: 2407-2412.

El Agha E, Herold S, Al Alam D, et al. Fgf10-positive cells represent a progenitor cell population during lung development and postnatally. Development 2014; 141: 296-306.

Li C, Li M, Li S, et al. Progenitors of secondary crest myofibroblasts are developmentally committed in early lung mesoderm. Stem Cells 2015; 33: 999-1012.

Mailleux AA, Kelly R, Velmaat JM. Fgf10 expression identifies parabronchial smooth muscle cell progenitors and is required for their entry into the smooth muscle cell lineage. Development 2005; 132: 2157-2166.

Moiseenko A, Kheirollahi V, Chao C-M, et al. Origin and characterization of alpha smooth muscle actin-positive cells during murine lung development. Stem Cells 2017; 35: 1566-1578.

Teisanu RM, Chen H, Matsumoto K, et al. Functional analysis of two distinct bronchiolar progenitors during lung injury and repair. Am J Respir Cell Mol Biol 2011; 44: 794-803.

B-S, Nolan DJ, Guo P, et al. Endothelial-derived angiocrine signals induce and sustain regenerative lung aveolarization. Cell 2011; 147: 539-553.

Rafii S, Butler JM, Ding B-S. Angiocrine functions of organ-specific endothelial cells. Nature 2016; 529: 316-325.

Rafii S, Cao Z, Lis R, et al. Platelet-derived SDF-1 primes the pulmonary capillary vascular niche to drive lung alveolar regeneration. Nat Cell Biol 2015; 17: 123-136.

Mulugeta S, Nureki S-I, Beers MF. Lost after translation: insights from pulmonary surfactant for understanding the role of alveolar epithelial dysfunction and cellular quality control in fibrotic lung disease. Am J Physiol Lung Cell Mol Physiol 2015; 309: L507-L525.

Young LR, Gulleman PM, Bridges JP, et al. The alveolar epithelium determines susceptibility to lung fibrosis in Hermansky-Pudlak syndrome. Am J Respir Crit Care Med 2012; 186: 1014-1024. Bhatia SN, Ingber DE. Microfluidic organs-on-chips. Nat Biotechnol 2014; 32: 760-772. proliferative population. Am J Physiol Lung Cell Mol Physiol 2002; 283: L1315-L1321.

Davidson DJ, Kilanowski FM, Randell SH, et al. A primary culture model of differentiated murine tracheal epithelium. Am J Physiol Lung Cell Mol Physiol 2000; 279: L766-L778.

Yoon JH, Gray T, Guzman K, et al. Regulation of the secretory phenotype of human airway epithelium by retinoic acid, triiodothyronine, and extracellular matrix. Am J Respir Cell Mol Biol 1997; 16: 724-731.

Huh D, Fujioka H, Tung Y-C, et al. Acoustically detectable cellular-level lung injury induced by fluid mechanical stresses in microfluidic airway systems. Proc Natl Acad Sci USA 2007; 104: 18886-18891.

Mahto SK, Tenenbaum-Katan J, Greenblum A, et al. Microfluidic shear stress-regulated surfactant secretion in alveolar epithelial type II cells in vitro. Am J Physiol Lung Cell Mol Physiol 2014; 306: L672-L683.

Trieu D, Waddell TK, McGuigan AP. A microfluidic device to apply shear stresses to polarizing ciliated airway epithelium using air flow. Biomicrofluidics 2014; 8: 064104.

Quintero NV, Song Y, Manneville P, et al. Behavior of liquid plugs at bifurcations in a microfluidic tree network Biomicrofluidics 2012; 6: 34105.

Song Y, Baudoin M, Manneville P, et al. The air-liquid flow in a microfluidic airway tree. Med Eng Phys 2011; 33: 849-856.

Baudoin M, Song Y, Manneville P, et al. Airway reopening through catastrophic events in a hierarchical network. Proc Natl Acad Sci USA 2013; 110: 859-864. $1662-1668$

Huh D, Leslie DC, Matthews BD, et al. A human disease model of drug toxicity-induced pulmonary edema in a lung-on-a-chip microdevice. Sci Transl Med 2012; 4: 159ra147.

Long C, Finch C, Esch M, et al. Design optimization of liquid-phase flow patterns for microfabricated lung on a chip. Ann Biomed Eng 2012; 40: 1255-1267.

Stucki AO, Stucki JD, Hall SRR, et al. A lung-on-a-chip array with an integrated bio-inspired respiration mechanism. Lab Chip 2015; 15: 1302-1310.

Blume C, Reale R, Held M, et al. Cellular crosstalk between airway epithelial and endothelial cells regulates barrier functions during exposure to double-stranded RNA. Immun Inflamm Dis 2017; 5: 45-56.

Sellgren KL, Butala EJ, Gilmour BP, et al. A biomimetic multicellular model of the airways using primary human cells. Lab Chip 2014; 14: 3349-3358.

Tenenbaum-Katan J, Fishler R, Rothen-Rutishauser B, et al. Biomimetics of fetal alveolar flow phenomena using microfluidics. Biomicrofluidics 2015; 9: 014120.

Fishler R, Sznitman J. A microfluidic model of biomimetically breathing pulmonary acinar airways. J Vis Exp 2016: e53588.

J Biomech 2013; 46: 2817-2823.

Fishler R, Hofemeier P, Etzion Y, et al. Particle dynamics and deposition in true-scale pulmonary acinar models. Sci Rep 2015; 5: 14071.

Benam $\mathrm{KH}$, Villenave R, Lucchesi $\mathrm{C}$, et al. Small airway-on-a-chip enables analysis of human lung inflammation and drug responses in vitro. Nat Methods 2016; 13: 151-157.

1 Barkal LJ, Procknow CL, Álvarez-García YR, et al. Microbial volatile communication in human organotypic lung models. Nat Commun 2017; 8: 1770.

Jain A, Barrile R, van der Meer AD, et al. Primary human lung alveolus-on-a-chip model of intravascular thrombosis for assessment of therapeutics. Clin Pharmacol Ther 2018; 103: 332-340. 
113 Takebe T, Zhang B, Radisic M. Synergistic engineering: organoids meet organs-on-a-chip. Cell Stem Cell 2017; 21: 297-300.

114 Benam KH, Novak R, Nawroth J, et al. Matched-comparative modeling of normal and diseased human airway responses using a microengineered breathing lung chip. Cell Syst 2016; 3: 456-466.

115 Douville NJ, Tung Y-C, Li R, et al. Fabrication of two-layered channel system with embedded electrodes to measure resistance across epithelial and endothelial barriers. Anal Chem 2010; 82: 2505-2511.

116 Nguyen TA, Yin T-I, Reyes D, et al. Microfluidic chip with integrated electrical cell-impedance sensing for monitoring single cancer cell migration in three-dimensional matrixes. Anal Chem 2013; 85: 11068-11076.

117 Liu M-C, Shih H-C, Wu J-G, et al. Electrofluidic pressure sensor embedded microfluidic device: a study of endothelial cells under hydrostatic pressure and shear stress combinations. Lab Chip 2013; 13: 1743-1753.

118 Williams MC. Alveolar type I cells: molecular phenotype and development. Annu Rev Physiol 2003; 65: 669-695.

119 Herzog EL, Brody AR, Colby TV, et al. Knowns and unknowns of the alveolus. Proc Am Thorac Soc 2008; 5: 778-782.

120 Weibel ER. On the tricks alveolar epithelial cells play to make a good lung. Am J Respir Crit Care Med 2015; 191: 504-513.

121 Takahashi K, Tanabe K, Ohnuki M, et al. Induction of pluripotent stem cells from adult human fibroblasts by defined factors. Cell 2007; 131: 861-872.

122 Takahashi K, Yamanaka S. Induction of pluripotent stem cells from mouse embryonic and adult fibroblast cultures by defined factors. Cell 2006; 126: 663-676. 